The Egyptian Journal of Biochemistry \& Molecular Biology VOL 38 (N.1 \& 2) 39-55 December. 2020

\title{
CYP2D6 EXPRESSION IS CUMULATIVELY UP REGULATED IN MULTIDRUG TREATED HEPATOMA CELLS: A PREDECTIVE PHARMACOGENETICS IN VITRO MODEL
}

\section{Sherine Khedr1* Thoria Donia1, Elsayed Ibrahim Salim,2 Mohamed Hessien 1}

\author{
${ }^{1}$ Division of Biochemistry, Department of Chemistry, Faculty of Science, \\ Tanta University, Egypt, \\ ${ }^{2}$ Department of Zoology, Faculty of Science, Tanta University, Egypt
}

Received 5/7/2020 - Accepted 23/8/2020

\begin{abstract}
Cancer and many other diseases require concomitant treatments with combinations of many drugs.Debrisoquine 4-Hydroxylayase (CYP2D6) is microsomal enzyme involved in phase I metabolism of a long list of drugs. Also, it is a marker of inter-individual variability in drug responsiveness. This study was designated to explore the regulation of CYP2D6 in hepatoma cells exposed to combinations of anticancer and epigenetic modifying drugs. HepG2 cell were treated with combinations of anticancer drug (Taxol), glucocorticoid (dexamethasone, DEX) and epigenetic modifiers: Trichostatin A (TSA) and 5 aza-deoxycytidine (5 aza-dC). The expression of CYP2D6 was determined by quantitative RT-PCR and compared to other CYPs and the corresponding cumulative apoptotic effect was determined by flow cytometry. The obtained results revealed thatUnder non-induced conditions, CYP2D6 was stably expressed and sub micromolar concentration of DEX mildly increased its expression. Individual treatments as DEX, Taxol, TSA and 5-azadC induced 2-6fold increase in the transcript level, where the TSA was the most potent inducer. Combinations of 2 drugs as (Taxol+DEX), (Taxol+TSA), (Taxol+5-aza-dC) and (TSA+5-aza-dC). led to 3-10fold increase (average 6.2), whereas cocktails of 3 drugs as $($ Taxol+DEX+5-aza-dC), (Taxol+DEX+TSA) and (Taxol+TSA+5-
\end{abstract}

${ }^{\star}$ Correspondence Aauthor (Sherine Khedr @hotmail.com) 
aza-dC) led to further up regulatory effect (11-27-fold). The highest increment (28-fold) was observed when cells were treated with 4 drugs as (Taxol+TSA+5-aza-dC+DEX). The progressive induction of CYP2D6 was correlated with the cumulative apoptotic effect $(r=0.79)$. Conclusively, the data suggest, for the first time, that anticancer, epigenetic regulatory factors and dexamethasone cumulatively enhanced the baseline expression of CYP2D6.

Keywords: 5-Aza deoxycytidine, CYPs, dexamethasone, Taxol,

Trichostatin A

\section{INTRODUCTION}

Treatment of cancer and many other pathological disorders usually require concomitant supplementation with combinations of many drugs, which are differentially metabolized by a long list of Cytochromes P450 (CYPs). These CYPs are a family of 60 members, widely distributed in different tissues and represent the main tool of phase I reactions involved in the biotransformation of drugs and other xenobiotics. The activity of CYPs is affected by their constitutive transcription factors, their genetic variability (polymorphism), and CYP-specific inducers or inhibitors. As liver is the main site of xenobiotics biotransformation, hepatic CYPs are influenced by modifiers including glucocorticoids(Matoulková et al., 2014), epigenetic factors and many other xenobiotics(Savas $\boldsymbol{e t}$ al., 1999). One-third of CYPs are reported to be under epigenetic control(Kacevska et al., 2011). In HepG2 cells, for example, inhibition of DNA methyltransferases (DNMTs) or histone deacetylase (HDACs) by 5 aza-2-deoxycytidine (5-aza-dC) and/or Trichostatin A (TSA) altered the expression of a large number of CYPs, suggesting the impact of epigenetic mechanisms on the regulation of CYPs(Dannenberg and Edenberg, 2006) . Also, CYPs play an important role in carcinogens and anti-cancer drug biotransformation. Among CYPs, Debrisoquine 4-Hydroxylayase (CYP2D6) has special importance, where it was introduced as a predictor of drug related responsiveness, inter-individual variation in addition to the relation between its polymorphism and the development of cancers and other pathological condition(Bezerra $\boldsymbol{e t}$ al., 2018; Singh et al., 2018). These observations relay on some unique features of CYP2D6 including its high genetic polymorphism 
with more than 130 allelic variants (databased in The Pharmacogenetic Variation (Nofziger et al., 2020), where it is the most investigated CYPs in relation to genetic polymorphism. Also, CYP2D6 protein has a wide activity range within a population that accounts the ultra-rapid metabolizers (UMs), extensive metabolizers (EMs), intermediate metabolizers (IMs) and poor metabolizers (PMs) phenotypes(Zhou, 2009). This increases the usability of CYP2D6 in clinical pharmacogenetic testing(Yang et al., 2017).

In liver, CYP2D6 is stably expressed and accounts for $<2 \%$ of the total hepatic CYPs and it mediates the metabolism in almost $25 \%$ of drugs(Yu et al., 2004; Zanger et al., 2004; Zhuge et al., 2004). HepG2 cells are predominantly used in the initial screening of drugs, the individual effects of many drugs and the involvement of different CYPs is well documented in the literature. Because CYP2D6 enzyme is responsible for the metabolism of antipsychotics, antidepressants, antiarrhythmics, and opioid analgesics(Eichelbaum et al., 2006), the impact of anticancer drugs on its transcription was ignored in the literature. Moreover, studies that focus on the implication of CYP2D6 in multi-drug interactions are sparse. Thus, this work is designated to investigate the impact of concomitant multi-drug treatments on CYP2D6 expression in hepatoma cells compared to other CYPs members subjected to similar conditions.

\section{MATERIAL AND METHOD}

5-Aza deoxycytidine (5-aza-dC) was purchased from Sigma-Aldrich (CAS No. 2353-33-65)., USA.Tricostatin A (TSA) was purchased fromSigma-Aldrich (CAS No. 58880-19-6)., USA. Dexamethasone was purchased from Amriya for pharmaceutical industries AlexandriaEgypt and Taxol, produced by Ebewa pharma (Cat number A-4866) was purchased from the local market. In all experiments, a fixed sub micromolar $(10 \mathrm{nM})$ of DEX was used. To induce histone deacetylation, cells were incubated with $5 \mathrm{mM}$ TSA, dissolved in dimethyl sulfoxide (DMSO) for 24 hrs.Hepatoma cells (HepG2) were generously provided by The Department of Cancer Biology, National Cancer Institute, Cairo University. Cell culture reagents Dulbecco's modified Eagle medium (DMEM), Fetal bovine serum (FBS) and penicillin, and streptomycin) were purchased from Lonza, BioWhittaker ${ }^{\circledR}$, USA. 


\section{Cell culture and treatment}

Cells were seeded in DMEM with L glutamine, supplemented with $10 \%$ FBS, $1 \%$ Penicillin and Streptomycin in a humidified atmosphere of $95 \%$ air and $5 \% \mathrm{CO}_{2}$ at $37{ }^{\circ} \mathrm{C}$. Initially, cells were cultured in a low cell density then passaged with defined cell densities according the experimental settings. Cells were cotreated with Taxol plus DEX and/or5-Aza-dC, TSA for 24(Gagandeep et al., 1999).Cells were cultured with initial cell density $3.5 \times 10^{5}$ cells $/ \mathrm{ml}$ in $100 \mu$ lof media containing increasing doses of Taxol plus DEX and/or 5-Aza-dC, TSA for $24 \mathrm{hr}$.In addition to the control plates, the study included 11 different drug treatments. In 4 treatment modes cells were exposed to a single drug (Taxol, DEX, TSA or 5 aza-dC). Other treatments included cells cotreated with combinations of 2,3 or 4 drugs.

Apoptotic assay:

In order to determine the apoptotic effect, Annexin V FITC kit (MiltenyiBiotec, Auburn, CA, USA) was used following the plates by scraping and centrifuged at $1000 \mathrm{rpm}$ for $5 \mathrm{~min}$. The cell was resuspended in $1 \mathrm{ml}$ PBS and then incubated with $0.25 \mu \mathrm{g} / \mathrm{ml}$ Annexin $\mathrm{V}$ reagent in $1 \mathrm{X}$ binding buffer for 15 min followed by two washes with Wash Buffer. Cells were resuspended in binding buffer containing $0.5 \mu \mathrm{g} / \mathrm{ml}$ propidium iodide (PI) then subjected to flow cytometry (BC Novus). The data was retrieved and analyzed by Kaluzasoftware(Gagandeep et al., 1999). All experiments were performed in triplicates where the average values were considered.

\section{Expression analysis by real-time quantitative PCR:}

The expression levels of CYP2C19 and CYP2D6 genes involved in drug-drug interaction were assessed using Qiagen Rotor-Gene QPCR Cycler 5 Plex. Total RNA was isolated using the GeneJET RNA purification kit, (ThermoFisher Scientific, USA) and $200 \mathrm{ng}$ was used for cDNA synthesis using SensiFAST ${ }^{\text {TM }}$ cDNA Synthesis Kit, (Bioline Inc, USA) following the manufacturer's protocol. $50 \mathrm{ng} / \mu \mathrm{l}$ $(2 \mu 1)$ of cDNA was used as a template in cycling reactions mixture $(20$ $\mu \mathrm{l})$ containing $50 \mathrm{nmol} / \mu \mathrm{l}(2 \mu \mathrm{l})$ of gene-specific primes, where the primers sequence of CYP2D6 sequence according to (Steijns and Van Der Weide, 1998) wereforward: 5'dATGAGAACCTGTGCATAG-3, reverse:

5'dCGGATGTAGGATCATGAG.3' and GAPDH sequence according to(Steijns and Van Der Weide, 1998)were forward: 5- 
CCTGCCAAGTATGATGACATCAAGA-3,reverse:5-

GTAGCCCAGG ATGCCCTTTAGT-3. For CYP2C19theforward: 5'dATTGAATGA AAACATCAGGATTG.3' and the reverse: 5'dGAGGGTTGTTGATGT CCATC.3'(Arefayene et al., 2003)

The reaction mix included 10 QIAGEN SYBR green, raised to $20 \mu \mathrm{l}$ with $\mathrm{ddH}_{2} \mathrm{O}$ then subjected a thermal cycling program consisted of a single step of initial denaturation and 45 cycles (each consists of single denaturation at $94{ }^{\mathrm{O}} \mathrm{C}$ for $5 \mathrm{sec}$, annealing at $67{ }^{\mathrm{o}} \mathrm{C}, 61{ }^{\mathrm{O}} \mathrm{C}$ (CYP2D6) and an extension step at $72{ }^{\circ} \mathrm{C}$ for $20 \mathrm{sec}$. Reactions were terminated with a single step in which the temperature was increased from $50{ }^{\circ} \mathrm{C}$ to $99{ }^{\circ} \mathrm{C}$ toensure the specificity of the amplicons and produce a melt curve. In parallel, the expression of GAPDH was used as an internal control to determine the fold expression changes in target genes. The critical threshold $(\mathrm{Ct})$ of target genes was normalized with quantities $(\mathrm{Ct})$ of GAPDH by using the $2^{-\Delta \Delta C t}$ to (Livak and Schmittgen, 2001)In qRT-PCR melt curves were justified to assess whether the intercalating dyes have produced product specific singles and to determine the $\mathrm{Ct}$ values.

\section{Statistical data analysis}

Apoptosis levels was measured and displayed as a percent of the untreated cells and represented as the mean of 3 runs \pm SD (standard deviation). The expression data were presented as fold expression relative to the GAPDH level. SPSS13.0 software package was used to analyze the data and Microsoft excel was used in graphing. Principle component analysis was performed with ClustVissoftwar.

\section{RESULTS}

\section{1-Determination of cell apoptosis.}

The apoptotic effects of Taxol, DEX, TSA and 5 aza-dC were investigated by flow cytometry in which treated cells were dually stained with Annexin V-FITC and Propidium iodide (PI). Figure 1 (panels A-C) demonstrates the scatter blots of flow cytometric analysis, where the percentages of cells that underwent early and late apoptotic fractions are represented in the lower and upper right quadrates, respectively, whereas the percent of viable and dead cells are shown in the lower and upper left quadrants, respectively. The 
obtained measurements were compared to the obtained values of the untreated cells as a reference sample (Table 1). The results demonstrated that the apoptosis was drug dependent, where it was significantly induced in cells individually treated with Taxol $(37.58 \%)$ or TSA (24\%), whereas both DEX and 5-aza-dC induced negligibleapoptotic effects. Treatment of cells with different drug combinations induced a variable apoptotic effect. This apoptotic effect was progressively increased along with increasing the number of drugs. The highest mean percentages of apoptosis were seen when cells were exposed to 3 or 4 drugs $(35.8 \%, 40.9 \%$ respectively) (Figure 2). The fluctuation seen in figure 2 due to the variability of the apoptotic effect with different drugs and different combinations.

A
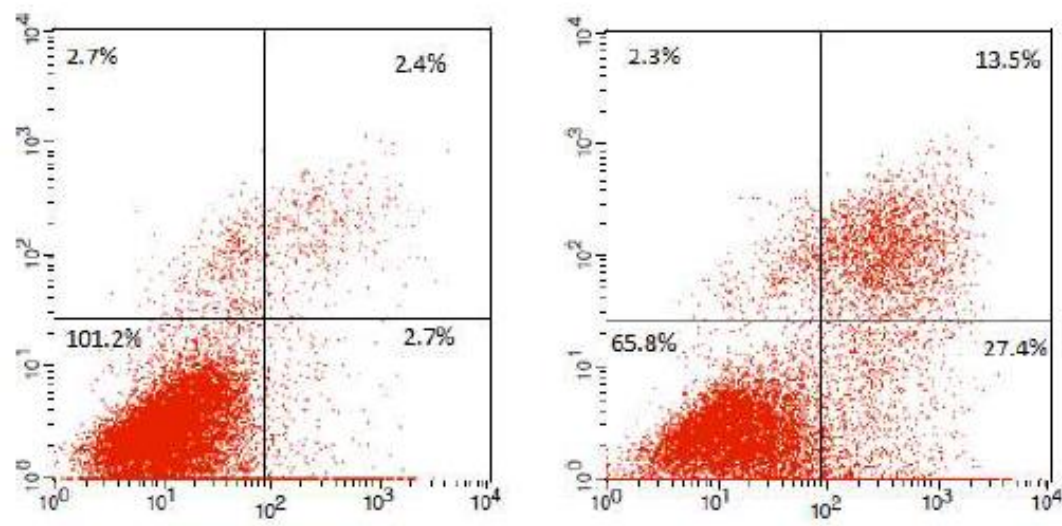

Dex

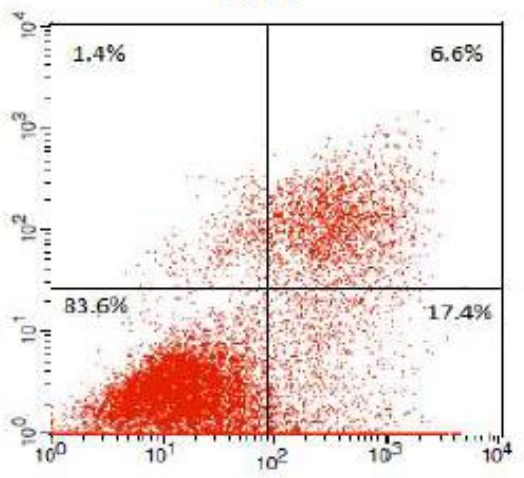

Taxol

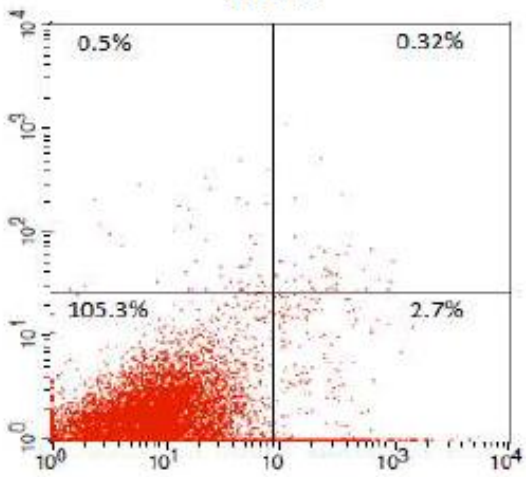

TSA

5 Aza-dC 


\section{B}
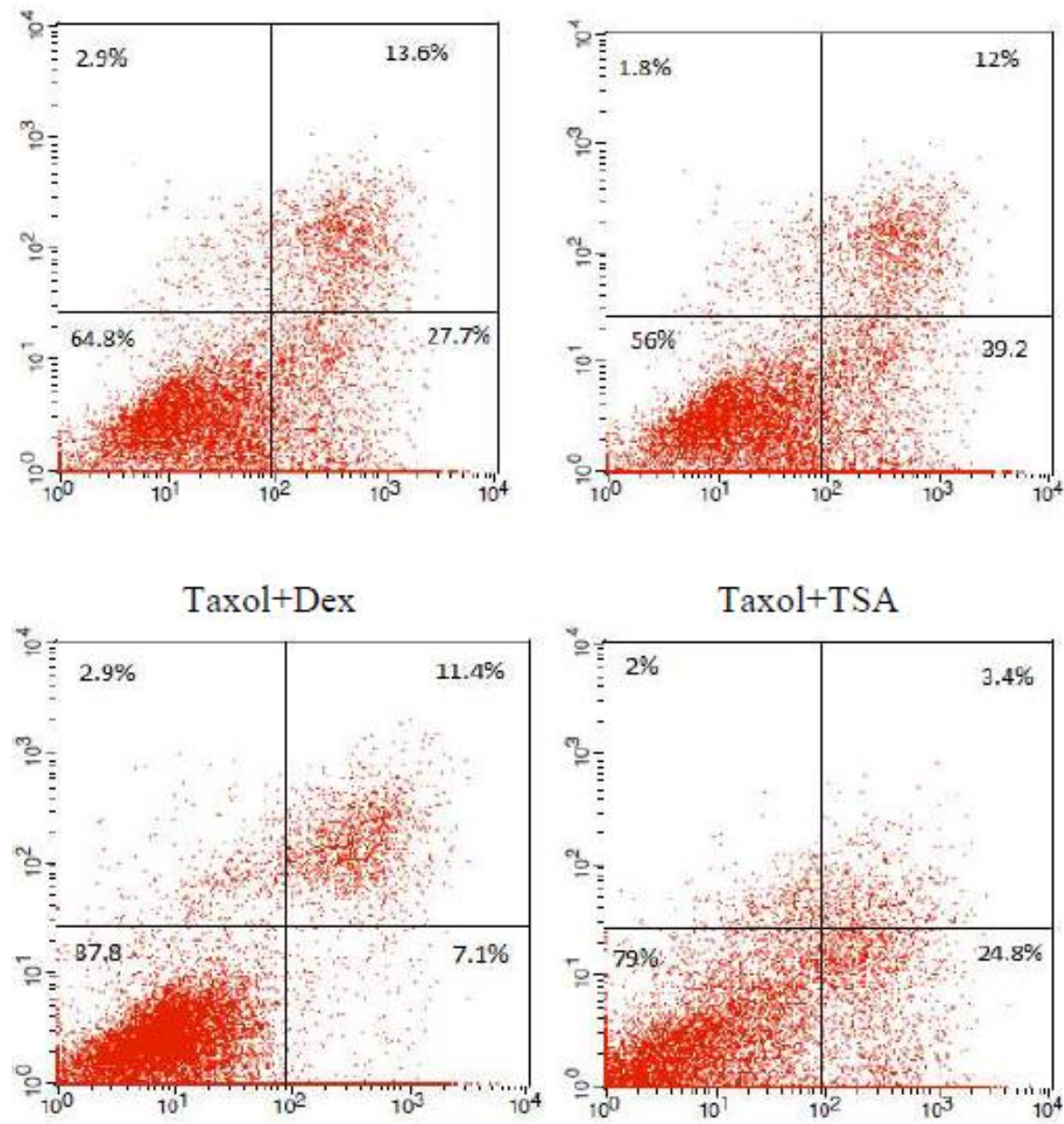

Taxol+5Aza-dC

$\mathrm{TSA}+5 \mathrm{Aza}-\mathrm{dC}$ 

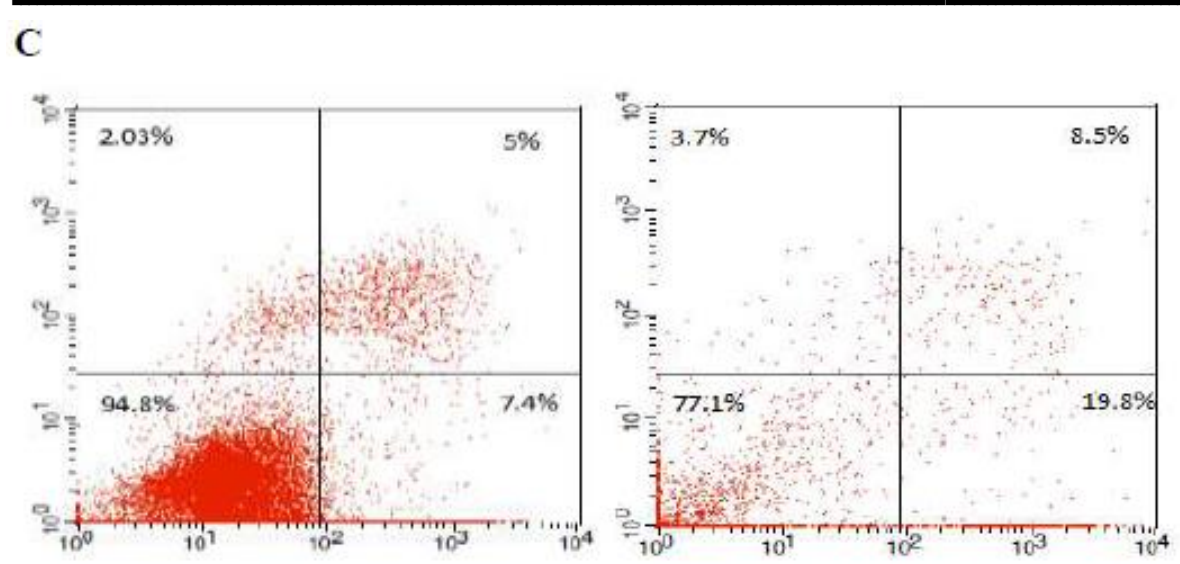

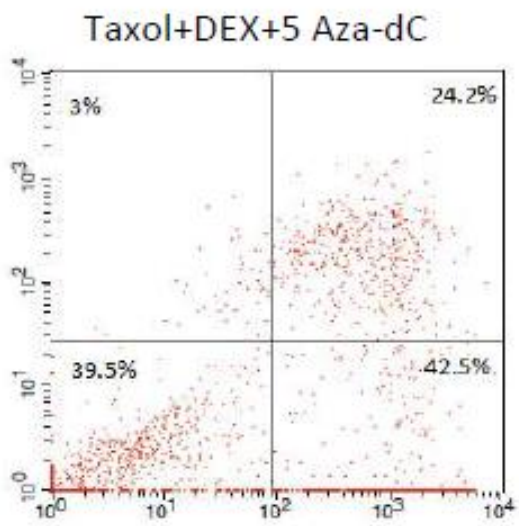

Taxol+TSA+5 Aza-dC

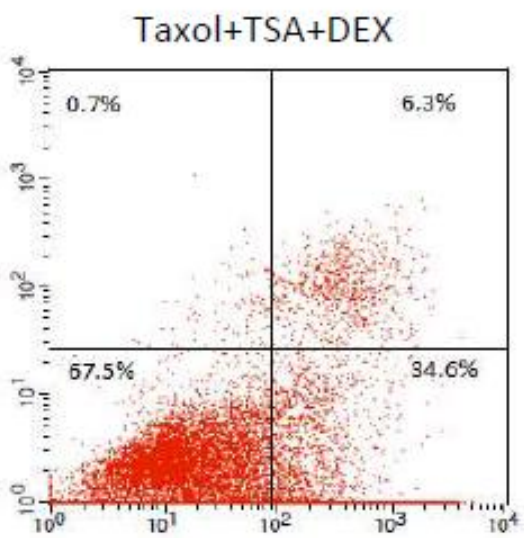

Taxol+TSA+5 Aza+ DEX

Figure 1. Apoptotic effect of different drugs combinations on HepG2 cells. Cells were treated with a single drug (A), combinations of 2 drugs (B), or combinations of 3 or 4 drugs (C). After incubation cells were stained with propidium iodide and subjected to flow cytometry to detect viable, early apoptotic, late apoptotic and dead cells shown in the lower left, lower right, upper right and upper left quadrants, respectively.

Abbreviations: Dex: dexamethasone, TSA: Trichostatin A, 5-aza-dC: 5-aza deoxycytidine. 
Table 1: Percent of apoptotic cells and CYP2D6 relative quantifications in different treatments.

\begin{tabular}{llc}
\hline Treatment & Apoptosis (\%) & RQs \\
\hline Dex & $5.1 \pm 1.76$ & $1.41 \pm 0.20$ \\
\hline Taxol & $40.9 \pm 0.41$ & $3.14 \pm 0.23$ \\
\hline TSA & $24 \pm 2.80$ & $6.23 \pm 0.48$ \\
\hline 5Aza-dC & $3 \pm 0.47$ & $1.97 \pm 0.27$ \\
\hline Taxol+Dex & $41.3 \pm 2.50$ & $4.56 \pm 0.48$ \\
\hline Taxol+TSA & $51.2 \pm 3.26$ & $6.41 \pm 0.55$ \\
\hline Taxol+5Aza-dC & $18.5 \pm 2.03$ & $2.94 \pm 0.65$ \\
\hline TSA+5 Aza-dC & $28.2 \pm 2.69$ & $10.7 \pm 0.63$ \\
\hline Taxol+DEX+5 Aza-D & $12.4 \pm 2.98$ & $11.47 \pm 0.49$ \\
\hline Taxol+TSA+DEX & $28.3 \pm 0.98$ & $11.63 \pm 0.72$ \\
\hline Taxol+TSA+5 Aza-dC & $66.7 \pm 6.17$ & $27.23 \pm 1.77$ \\
\hline Taxol+TSA+5 Aza+DEX & $40.9 \pm 5.96$ & $28.25 \pm 1.96$ \\
\hline
\end{tabular}
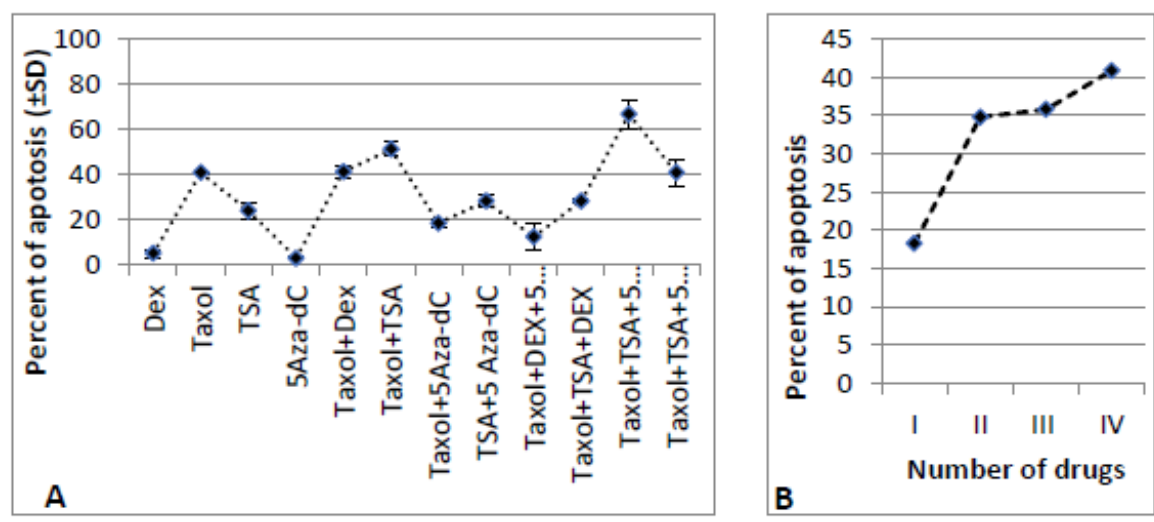

Figure 2. Variability of the apoptotic effects of combinations of Taxol, DEX, TSA and 5-aza-dC in HepG2 cells (A). In single treatments the apoptotic effect was drug dependent. Combinations of drugs induced a relative increase, where the highest percentage of the apoptotic cells is observed in cells exposed to cocktails of Taxol and/or TSA (D). Maximum apoptosis is seen in cells treated with 3 or 4 drugs (B). 


\section{2-Expression analysis}

The expression analysis of CYP2D6 was determined in cells exposed to deferent single or multidrug treatments, where it was estimated as an expression fold relative to the GAPDH expression. Single treatments induced CYP2D6 expression following the order TSA $>$ Taxol $>5$-az dC $>$ DEX) (Figure 2, panel A). Combinations of 2 drugs led to 3-10-fold increase (average 6.2), whereas cocktails of 3 drugs induced more transcription (11-27-fold, mean 16.8). The highest increment (28-fold) was observed when cells were treated with 4 drugs (Figure 3, Table 1). Furthermore, the progressive induction of CYP2D6 was correlated with the corresponding cumulative apoptotic effect ( $\mathrm{r}=0.79)$ (Figure 4). To investigate the mutual correlations between different combinations, principle component analysis was performed. Treatments with 2,3 and 4 drugs were clustered with limited distances, whereas cells population treated with 4 drugs did not pool with any of the other overlapping clusters.

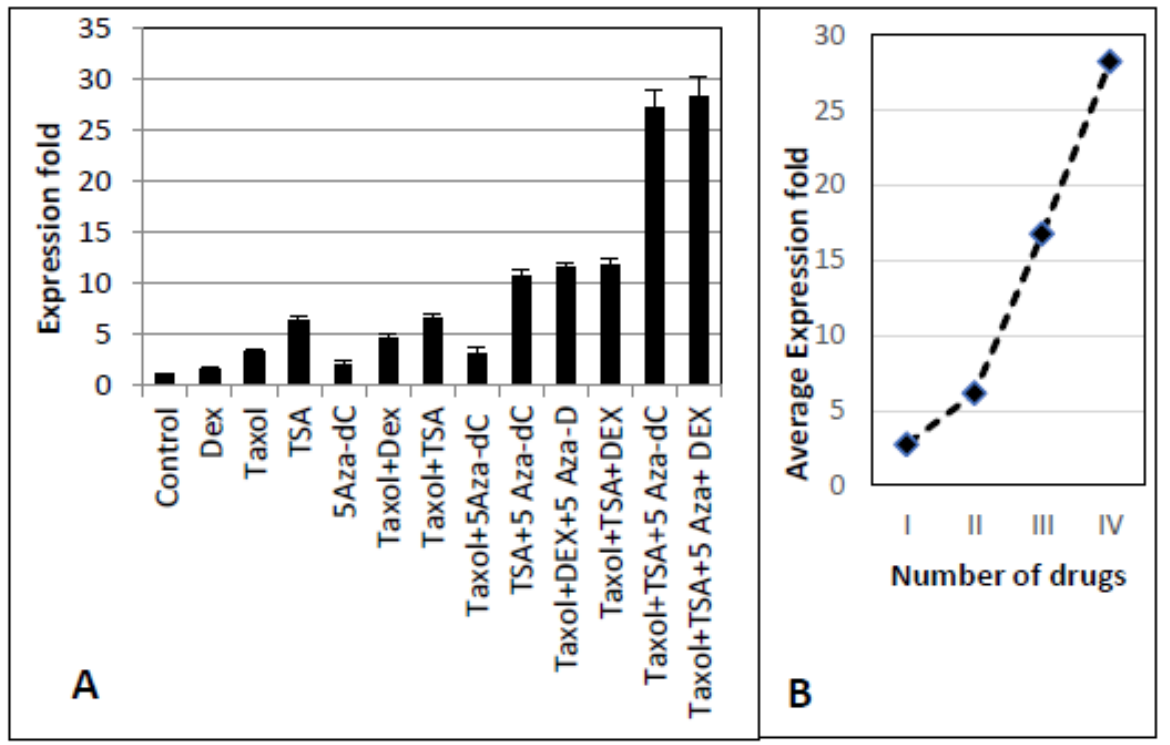

Figure 3. Changing pattern of CYP2D6 expression in hepatoma cells. (A) Cells were treated with different combinations of Taxol, DEX, TSA and 5-aza-dC (A). (B) The average increase of the gene transcripts when cells were treated with a single drug or concomitantly treated with 2,3 or 4 drugs. 


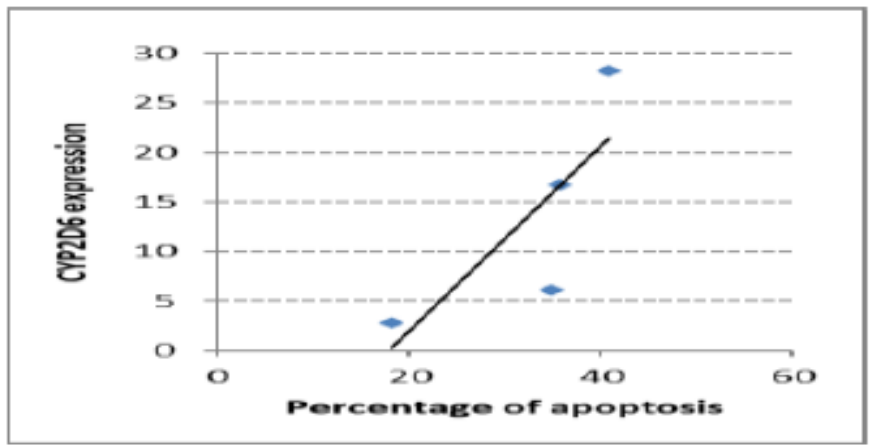

Figure 4: Correlation between apoptosis and CYP2D16 expression showing a cumulative apoptotic effect of Taxol, DEX, TSA and 5-aza$\mathrm{dC}$ is correlated with their induction of CYP2D expression (Person correlation $\mathrm{r}=0.79)$.

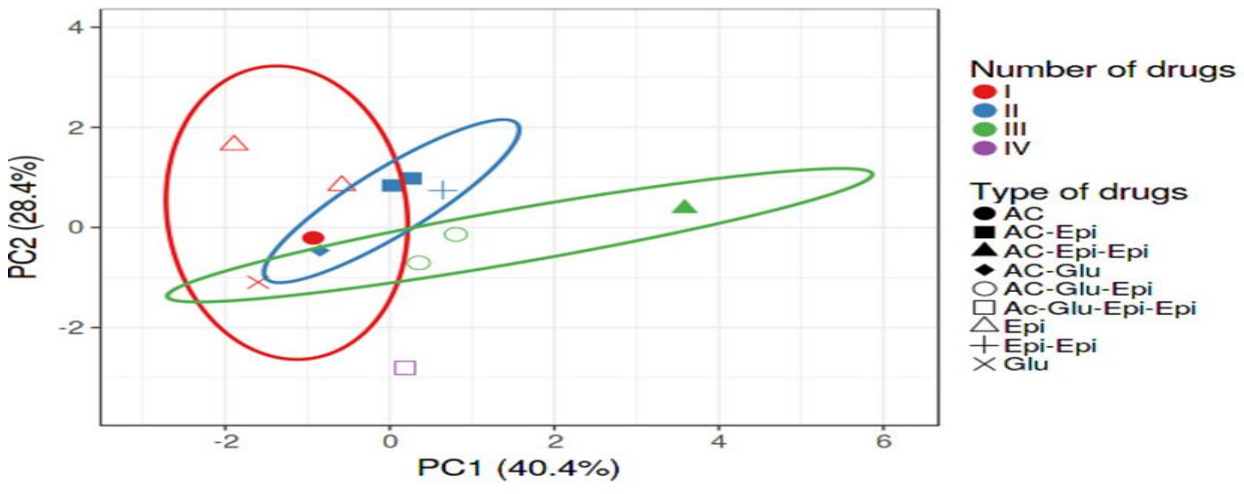

Figure 5:Principle components analysis of the expression biplot of CYP2D6 and 4 other CYPs genes (1A2,2B6,3A4,2C19) differentially expressed in HepG2 cells treated in 12 modes. $X$ and $Y$ axis show principal component 1 (PC1) and principal component 2 (PC2) that explain $40.4 \%$ and $28.4 \%$ of the total variance, respectively. Prediction ellipses demonstrates the clustering of cells treated with 1,2 or 3 drugs. Cells treatment with 4 drugs is represented by hollow squire.

\section{DISCUSSION}

The metabolic activity of CYP2D6 varies greatly between individuals, influencing dosing, efficacy and toxicity of CYP2D6metabolized drugs(Gaedigk $\boldsymbol{e t}$ al., 2008).This reflects the wide range of its expression among individuals. Moreover, this gene is considered one of the most polymorphic CYPs. These characteristics qualified 
CYP2D6 as a pharmacogenetics marker in some cancers (Chan et al.,2020). Herein, the study monitored the expression of CYP2D6 in hepatoma cells exposed to combinations of short list of drugs (2 to 4), including the anticancer drug (Taxol) predominantly used in treatment of many cancers, DEX, DNA methyltransferase inhibitor (5-aza-dC) and histone deacetylase inhibitor (TSA). This drug panel does not include any of the known CYP2D6 inhibitors. Also, no drug-drug interaction was reported among them. CYP2D6 was widely considered to be non-inducible on the basis of hepatocyte studies. However, other in vivo data suggested its induction by both endo-and xenobiotics (Farooq et al., 2016). The data presented here may challenge the previously reported studies that indicated the none or modest inducibility of CYP2D6 in most commonly used in vitro system of human hepatocytes(Dixit et al., 2007). The baseline expression of CYP2D6 mildly increased (1.4-fold) in cells treated with $10 \mathrm{nM}$ DEX for $24 \mathrm{~h}$, the observation was previously reported in HepaRG cells treated with higher concentrations of DEX for a longer period(Pan et al., 2017). The therapeutic use of DEX is well documented, where it reduced inflammation, used with other drugs to treat some types of cancers and cancer associated complications. In addition to DEX, the other drugs we used have a well-established anticancer effect (Taxol) or have potential anticancer role mediated through the epigenetic modulations such as TSA (Chen et al., 2019)and 5-aza-dC (Tao et al., 2012). Theyvariably induced 3, 6 and 2-fold increase in CYP2D6 transcription, respectively. Furthermore, co-treatments with different combinations (2-4 drugs) revealed progressive enhancement of the transcription. These data may present a newly developed observation, because before 2015 candidate anticancer drugs were not screened for putative CYP2D6 induction nor mandated by regulatory agencies including US Food and Drug Administration (FAD). Later, FDA included CYP2D6 for use in index clinical drug-druginteraction(DDI) studies (Grimstein et al., 2019). In human pluripotent stem cells-derived hepatocytes (hESCHep), the mRNA levels of CYP2D6 was much lower than in primary hepatocytes (hPH) due to the hypermethylation of $\mathrm{CpG}$ islands of CYPs, whereas the enhanced expression of CYPs in primary hepatocytes (hPH) was consistent hypomethylation of the $\mathrm{CpG}$ sites and permissive histone modifications. Accordingly, we observed that treatment with HDAC inhibitor (TSA) or DNMT inhibitor (5-aza-dC), 
upregulated CYP2D6 by 6.3 and 1.97 folds, respectively. Combined treatment with both modifiers, further enhanced the transcript level to 10.7-fold. To validate this observation, the expression of a short list of other CYPs (CYP1A2, CYP3A4, CY2B6 and CYP2C19) was investigated under similar culture conditions. None of these genes showed cumulative response as CYP2D6. The inhibition of DNMTs and HDACs is translated into an inhibition of methylation and increase histone acetylation, respectively leading to the observed upregulation of their transcript. This may agree with previous reports in which co-inhibition of DNMTs and HDACs increased the transcription of CYP2D6 and other CYPs members (Ru et al., 2018). As CYP2D6is controlled bysmall heterodimer partner (SHP) and Krüppel-like factor 9 (KLF-9)(Pan et al., 2017), as a transcriptional repressor and an activator, respectively, the epigenetic mediated upregulation of Klf-9 may explain the indirect effect on CYP2D6. Other scenarios may involve CYP2D6 copy number, especially populations studies have documented the high copy number variation (CNVs) of CYP2D6 relative to other CYPs(Ramírez et al., 2019). Although CYP2D6 copy number in HepG2 cells was not identified, previous data showed that increasing the copy numbers in liver biopsy tended to increase up to 5 copies leading to the higher expression level of CYP2D6 but not for patients with more than 5 copies (Wang et al., 2011). CYP2C19, which is closely related with CYP2D6 indrugtherapy of depression, gastroesophageal reflux and cardiovascular diseases, the gene did not show similar changing pattern to the CYP2D6. Surprisingly, comparative analysis demonstrated a good correlation $(\mathrm{r}=0.79)$ between the average apoptotic effects and the corresponding expression of CYP2D6.

Although the expression data in this work is limited (60 data points: generated from 5 genes in 12 treatment modes), principle component analysis was employed to depict the overall behavior of cells under multidrug treatments. Cumulative data analysis demonstrated that cells treated with 4 drugs did not pool inside any of the prediction ellipses of cells treated with a single, 2 or 3 drugs. Furthermore, no observable clear distinctive clustering was found based on number of drugs as indicated by the overlapping ellipses areas.

Conclusively, the data presents HepG2 cells as an in vitro pharmacogenetics toll in which CYP2D6 expression is stably 
expressed and progressively induced by anticancer (Taxol), glucocorticoids (DEX) and epigenetic modifiers (TSA and 5-aza-dC). More hepatic cell lines and in vivo studies are required to validate the implications for CYP2D6 in drug-drug interactions, drug metabolizing potential and screening of other anticancer and epigenetic modifiers.

Author Contributions: Conceptualization: M.H; Methodology and investigations, S.K and T.D. Supervision, review and editing, E.I.S; Writing original draft and correspondence, M.H.

\section{REFERENCE}

Arefayene, M., Skaar, T. C., Zhao, X., Rae, J. M., Tanus-Santos, J. E., Brinkmann, U., Brehm, I., Salat, U., Nguyen, A. and Desta, Z. (2003):Sequence diversity and functional characterization of the 5 'regulatory region of human CYP2C19. Pharmacogenetics and Genomics, 13(4), 199-206.

Bezerra, L. S., Santos-Veloso, M. A. O., Junior, N. d. S. B., da Fonseca, L. C. and Sales, W. L. A. (2018):Impacts of Cytochrome P450 2D6 (CYP2D6) genetic polymorphism in Tamoxifen therapy for breast cancer. Revista Brasileira de Ginecologia e Obstetrícia/RBGO Gynecology and Obstetrics, 40(12), 794-799.

Chan, C., Law, B., So, W., Chow, K. M., \& Waye, M. (2020).Pharmacogenomics of breast cancer: highlighting CYP2D6 and tamoxifen. Journal of cancer research and clinical oncology, 146(6), 1395-1404.

Chen, J. C. H., Chuang, H. Y., Liao, Y. J., Hsu, F. T., Chen, Y. C., Wang, W. H. and Hwang, J. J. (2019):Enhanced cytotoxicity of human hepatocellular carcinoma cells following pretreatment with sorafenib combined with trichostatin A. Oncology Letters, 17(1), 638645.

Dannenberg, L. O. and Edenberg, H. J. (2006):Epigenetics of gene expression in human hepatoma cells: expression profiling the response to inhibition of DNA methylation and histone deacetylation. BMC genomics, 7(1), 181.

Dixit, V., Hariparsad, N., Desai, P. and Unadkat, J. D. (2007):In vitro LC- MS cocktail assays to simultaneously determine human cytochrome P450 activities. Biopharmaceutics \& drug disposition, 28(5), 257-262. 
Eichelbaum, M., Ingelman-Sundberg, M. and Evans, W. E. (2006):Pharmacogenomics and individualized drug therapy. Annu. Rev. Med., 57, 119-137.

Farooq, M., Kelly, E. J. and Unadkat, J. D. (2016):RETRACTION: CYP2D6 Is Inducible by Endogenous and Exogenous Corticosteroids. Drug Metabolism and Disposition, 44(5), 750-757.

Gaedigk, A., Simon, S., Pearce, R., Bradford, L., Kennedy, M. and Leeder, J. (2008):The CYP2D6 activity score: translating genotype information into a qualitative measure of phenotype. Clinical Pharmacology \& Therapeutics, 83(2), 234-242.

Gagandeep, S., Novikoff, P. M., Ott, M. and Gupta, S. (1999):Paclitaxel shows cytotoxic activity in human hepatocellular carcinoma cell lines. Cancer letters, 136(1), 109-118.

Grimstein, M., Yang, Y., Zhang, X., Grillo, J., Huang, S.-M., Zineh, I. and Wang, Y. (2019):Physiologically based pharmacokinetic modeling in regulatory science: an update from the US Food and Drug Administration's Office of Clinical Pharmacology. Journal of pharmaceutical sciences, 108(1), 21-25.

Kacevska, M., Ivanov, M. and Ingelman- Sundberg, M. (2011):Perspectives on epigenetics and its relevance to adverse drug reactions. Clinical Pharmacology \& Therapeutics, 89(6), 902-907.

Livak, K. J. and Schmittgen, T. D. (2001):Analysis of relative gene expression data using real-time quantitative PCR and the 2- $\Delta \Delta \mathrm{CT}$ method. methods, 25(4), 402-408.

Matoulková, P., Pávek, P., Malý, J. and Vlček, J. (2014):Cytochrome P450 enzyme regulation by glucocorticoids and consequences in terms of drug interaction. Expert opinion on drug metabolism \& toxicology, 10(3), 425-435.

Nofziger, C., Turner, A. J., Sangkuhl, K., Whirl- Carrillo, M., Agúndez, J. A., Black, J. L., Dunnenberger, H. M., Ruano, G., Kennedy, M. A. and Phillips, M. S. (2020):PharmVar GeneFocus: CYP2D6. Clinical Pharmacology \& Therapeutics, 107(1), 154-170.

Pan, X., Ning, M. and Jeong, H. (2017):Transcriptional regulation of CYP2D6 expression. Drug Metabolism and Disposition, 45(1), 42-48. Ramírez, B., Niño-Orrego, M. J., Cárdenas, D., Ariza, K. E., Quintero, K., Bravo, N. C. C., Tamayo-Agudelo, C., González, M. A., Laissue, P. and Mendoza, D. J. F. (2019):Copy number variation profiling in 
pharmacogenetics CYP-450 and GST genes in Colombian population. BMC medical genomics, 12(1), 110.

Ru, B., Sun, J., Tong, Y., Wong, C. N., Chandra, A., Tang, A. T. S., Chow, L. K. Y., Wun, W. L., Levitskaya, Z. and Zhang, J. (2018):CR2Cancer: a database for chromatin regulators in human cancer. Nucleic acids research, 46(D1), D918-D924.

Savas, Ü., Griffin, K. J. and Johnson, E. F. (1999):Molecular mechanisms of cytochrome P-450 induction by xenobiotics: An expanded role for nuclear hormone receptors. Molecular Pharmacology, 56(5), 851-857.

Singh, H., Lata, S. and Gangakhedkar, R. R. (2018):Prevalence of CYP 2D6* 4 1934G/A polymorphism in Western Indian HIV patients. Apmis, 126(11), 842-851.

Steijns, L. S. and Van Der Weide, J. (1998):Ultrarapid drug metabolism: PCR-based detection of CYP2D6 gene duplication. Clinical chemistry, 44(5), 914-917.

Tao, S.-F., Zhang, C.-S., Guo, X.-L., Xu, Y., Zhang, S.-S., Song, J.R., Li, R., Wu, M.-C. and Wei, L.-X. (2012):Anti-tumor effect of 5aza-2'-deoxycytidine by inhibiting telomerase activity in hepatocellular carcinoma cells. World journal of gastroenterology: WJG, 18(19), 2334.

Wang, D., Jiang, Z., Shen, Z., Wang, H., Wang, B., Shou, W., Zheng, H., Chu, X., Shi, J. and Huang, W. (2011):Functional evaluation of genetic and environmental regulators of p450 mRNA levels. PloS one, 6(10), e24900.

Yang, Y., Botton, M. R., Scott, E. R. and Scott, S. A. (2017):Sequencing the CYP2D6 gene: from variant allele discovery to clinical pharmacogenetic testing. Pharmacogenomics, 18(7), 673-685. Yu, A. M., Idle, J. R. and Gonzalez, F. J. (2004):Polymorphic cytochrome P450 2D6: humanized mouse model and endogenous substrates. Drug metabolism reviews, 36(2), 243-277.

Zanger, U. M., Raimundo, S. and Eichelbaum, M. (2004):Cytochrome P450 2D6: overview and update on pharmacology, genetics, biochemistry. Naunyn-Schmiedeberg's archives of pharmacology, 369(1), 23-37.

Zhou, S.-F. (2009):Polymorphism of human cytochrome P450 2D6 and its clinical significance. Clinical pharmacokinetics, 48(12), 761804. 
Zhuge, J., Yu, Y.-N. and Wu, X.-D. (2004):Stable expression of human cytochrome P450 2D6* 10 in HepG2 cells. World Journal of Gastroenterology, 10(2), 234.

\section{الملخص العربي}

زيادة التعبير الجينى لجين انزيم CYP2D6 بثكل تراكمى فى خلايا الورم الكبدي المعالج

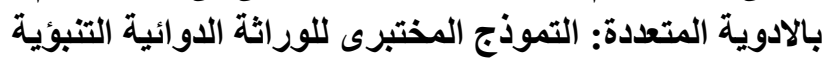

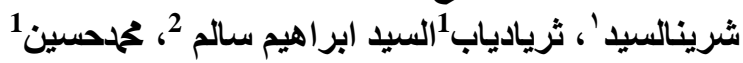

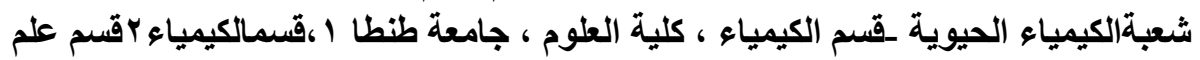

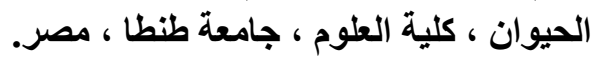

يستلزم علاج السرطان وظروف باثولوجية أخرى التناول المتزامن لعدة عقاقير .ويعد إنزيم

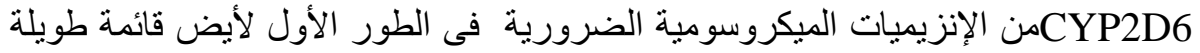

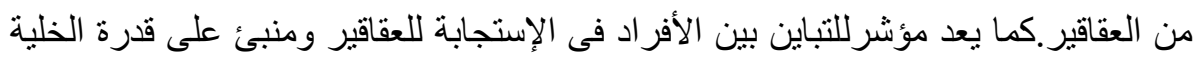

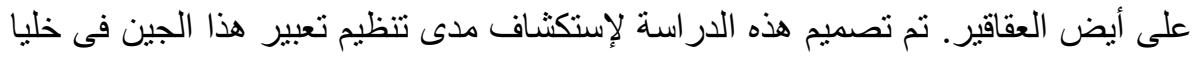

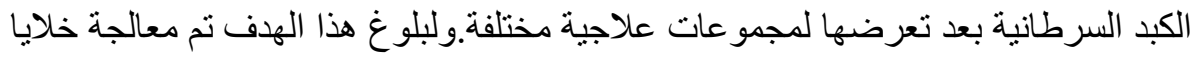

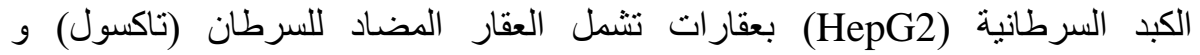

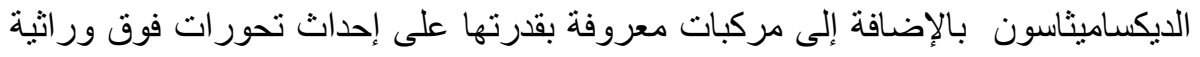

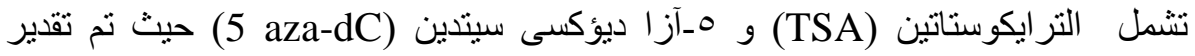

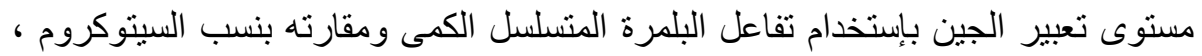

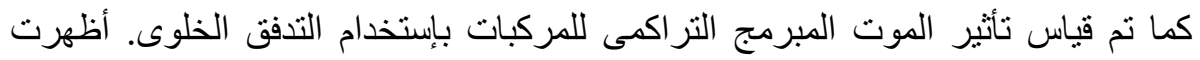

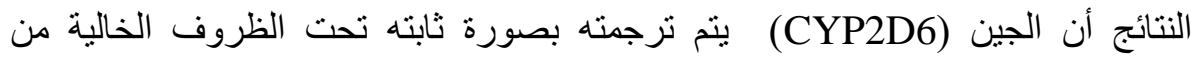

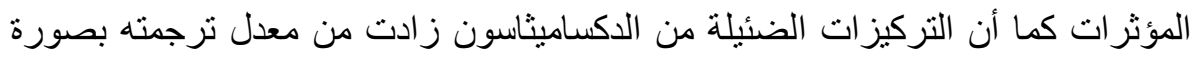

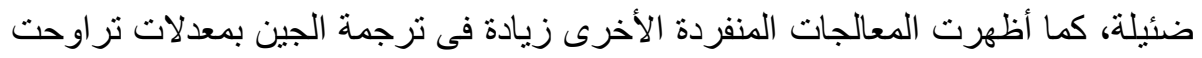

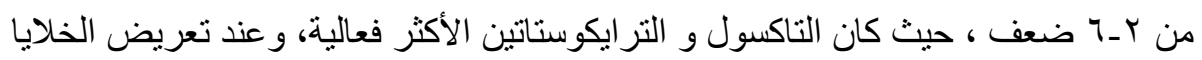

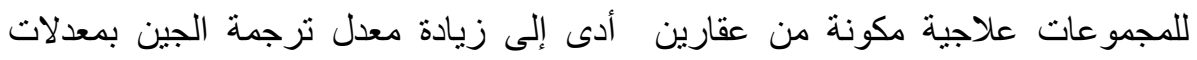

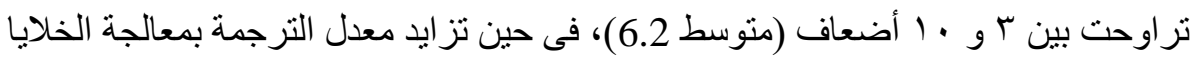

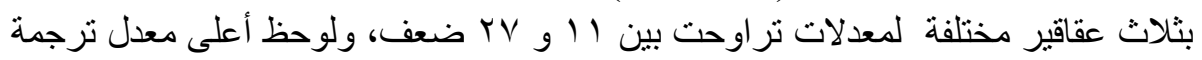

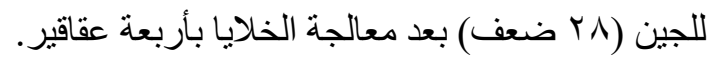

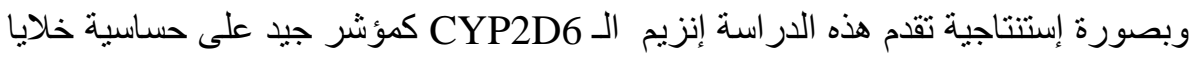

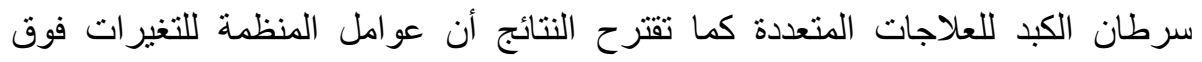

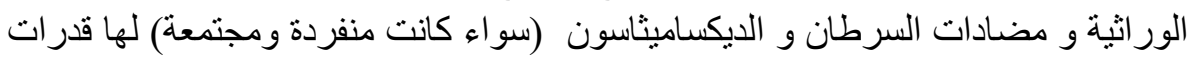
متباينة على زيادة ترجمة الجين. 